\title{
Nonlinear analysis of shape memory alloy beam under the thermal and the mechanical loads
}

\author{
Jichang Wang ${ }^{1}$, Jingning Yang ${ }^{1}$
}

\footnotetext{
${ }^{1}$ School of Science, Lanzhou University of Technology, 730050, Lanzhou, Gansu, China. e-mail: jichangw@163.com, yangjn@lut.cn.
}

\begin{abstract}
In this paper, the tension-compression asymmetry of shape memory alloys (SMA), subjected to the thermal and the mechanical loads, is sufficiently explored. Taking a basis of stress-strain relationship and a concern for critical phase transformation, a new simple model of mechanics is established. Through the deflection lines under different load conditions and the maximum deflection-bending moment curve, the effects of material nonlinearity and geometric nonlinearity on bending deformation of SMA beam are investigated. The results show that the neutral layer offset is related to the tension compression asymmetry coefficient and temperature. The phase transformation of SMA beam becomes more and more difficult as the increase of tension compression asymmetry coefficient and temperature. For the earlier phase transformation, material nonlinearity and geometric nonlinearity have little influence on beam deflection, but great in the late phase transformation.
\end{abstract}

Keywords: Shape memory alloy, nonlinear, tension-compression asymmetry, temperature

\section{INTRODUCTION}

SMA have been receiving increasingly attention and studied since the discovery in 1960s. Now, SMA have entered a wide range of important areas such as aerospace, machinery, civil engineering and medical [1-6]. These alloys present two special behaviors: superelastic effect and shape memory effect. SMA materials occur bending deformation under the external loads, which made the austenite change into martensite. The relationship between stress and strain is nonlinear in the process of phase transformation, which made SMA possess superelastic effect. And the shape memory effect can make the material recover its pristine shape after a residual strain by heating the material [7-10].

Many researchers have further studied on SMA and established multiple mechanical models in order to show the theory of phase transformation of SMA. Muller et al. [11] developed a constitutive model of SMA that had a vital effect on studying its mechanical constitutive model. Tanaka and Liang [12,13] established a new constitutive model with phenomenological theory, which presented a strong applicability for engineering. Bajoria et al. [14] used finite element method to analysis the bending deformation behavior of concrete beam using SMA. It found that the SMA beam has strong bearing capacity and large deformation. Atanakovic et al. [15] analyzed the pure bending deformation of SMA beams, and the expression of stress distribution along the height of the cross section was obtained. Some numerical simulation and experimental analysis of bending deformation of SMA wires were exhibited by Flor et al. [16]. They had a consideration about tension-compression asymmetry and used a numerical scheme to calculate the bending response. In recent years, based on complex constitutive model and the assumption of same properties between tension and compression, Eshghinejad [17] studied the bending deformation of SMA beam. Their model was developed for both loading and unloading phase. The most of existing studies, based on the assumption of the tension-compression symmetry, explored the superelastic effect and shape memory effect of SMA beam. Although some researchers considered the different properties between tension and compression, the model was complex. Even they ignored the problem of different properties between tension and compression of SMA beam, under the thermal and the mechanical loads.

The approach is based on the large deformation theory of beams and aims at solving the different properties between tension and compression of SMA materials. The tension compression asymmetry coefficient $\alpha$ [18] is used to amend original mechanical model. Considering the relationship between temperature and critical stress, the split-step method is adopted to analyze the effect of $\alpha$ on the SMA beam. Then, we 
establish the expression of beam cross section stress, martensitic volume fraction, bending moment and deflections. The nonlinear governing equation for SMA beam is derived. Finally, we discuss the effect of material nonlinearity and geometric nonlinearity on bending deformation of SMA beam.

\section{NONLINEAR BENDING DEFORMATION OF SMA BEAM}

\subsection{The mechanical model of SMA beam}

As can be seen in Figure 1, the length of SMA beam with rectangular cross section is $l, b$ and $h$ are the width and half of the height, respectively. In the process of bending deformation of SMA beam, the distance of neutral axis to tension side edge is $h_{1}$ and to compression side edge is $h_{2}$, under the bending moment $M$.

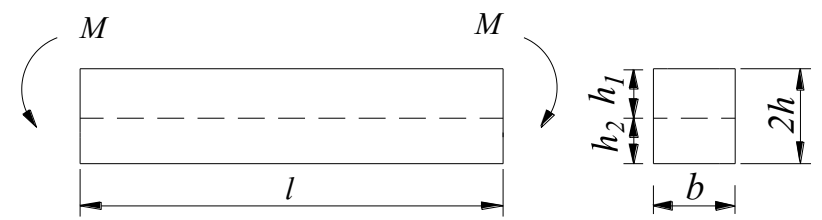

Figure 1: The mechanical model of SMA beam

\subsection{The simplified constitutive model of stress-strain}

Assuming the Young's moduli in mixed phase is constant, the simplified constitutive model of stress-strain is obtained as shown in Figure 2.

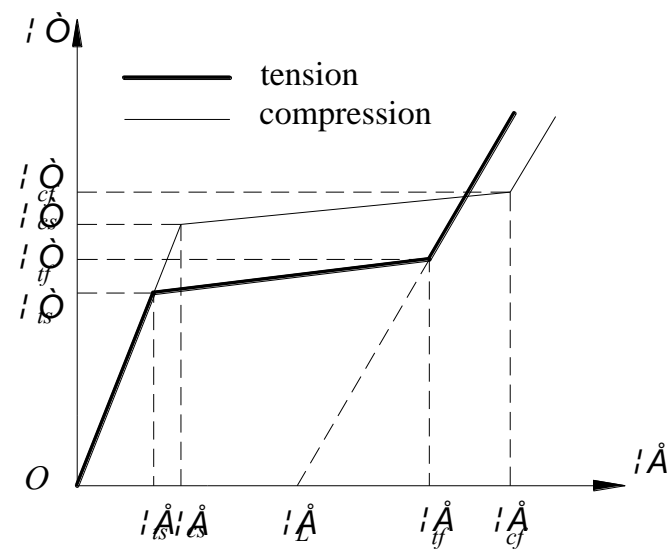

Figure 2: The simplified constitutive model of stress-strain

In the above figure, $\sigma_{t s}, \sigma_{t f}, \sigma_{c s}$ and $\sigma_{c f}$ are the stresses at the beginning and end of phase transformation on the tension side and beginning and end of phase transformation on the compression side, respectively. $\varepsilon_{t s}, \varepsilon_{t f}, \varepsilon_{c s}$ and $\varepsilon_{c f}$ are the strain at the beginning and end of phase transformation on the tension side and beginning and end of phase transformation on the compression side, respectively. $\varepsilon_{L}$ is the maximum residual strain. The used critical stresses are dependent on temperature. This relationship is illustrated in the phase plot shown in Figure 4.

According to the basic idea of continuum mechanics, SMA beam meets the plane assumption in the process of bending deformation. The strain on the surface of tension side and compression side of SMA beam can be expressed as Eq. (1)

$$
\varepsilon_{t}=\frac{h_{1}}{\rho} \quad \varepsilon_{c}=\frac{h_{2}}{\rho}
$$

The strain at the beginning of phase transformation on the tension side can be expressed as Eq. (2) 


$$
\varepsilon_{t s}=\frac{\sigma_{t s}}{E_{A}}
$$

The strain at the end of phase transformation on the tension side can be expressed as Eq. (3)

$$
\varepsilon_{t f}=\varepsilon_{L}+\frac{\sigma_{t f}}{E_{M}}
$$

In the above equations, $\rho, E_{A}$ and $E_{M}$ are the radius of curvature, Young's moduli in austenite and Young's moduli in martensite.

\subsection{Tension compression asymmetry coefficient}

The stress and the strain of original mechanical model are amended by introducing $\alpha$. Eq. (4) defines the relationship between $\alpha$ and the stress on tension side and compression side.

$$
\alpha=\frac{\sigma_{c s}-\sigma_{t s}}{\sigma_{c s}+\sigma_{t s}}=\frac{\sigma_{c f}-\sigma_{t f}}{\sigma_{c f}+\sigma_{t f}}
$$

\subsection{Stress strain relationship}

With the increase of bending moment, SMA beam undergoes a process from elastic deformation to phase transformation. The process of bending deformation of SMA beam can be divided into two stages: initial stage and phase transformation stage.

2.4.1 Initial stage $\left(\varepsilon_{t} \leq \varepsilon_{t s}\right)$

In the initial stage, the maximum strain does not reach the strain at the beginning of phase transformation, the SMA beam is total in austenite. The expression of stress distribution along the height of the cross section can be expressed as Eq. (5)

$$
\sigma(y)=\frac{E_{A} y \varepsilon_{t}}{h} \quad-h \leq y \leq h
$$

\subsubsection{Phase transformation stage $\left(\varepsilon_{t} \geq \varepsilon_{t s}\right)$}

As can be seen in Figure 3, when the maximum strain reach the strain at the beginning of phase transformation, the phase transformation from austenite to martensite occurs and the neutral axis moves to the compression side. A, M, and AM are the austenite, martensite and mixed phase, respectively. $\Delta_{i} h$ is the neutral axis offset in different stages. It is shown in Figure 3(a), for $\left|\varepsilon_{c}\right| \leq \varepsilon_{c s}$ and $\varepsilon_{t s} \leq \varepsilon_{t} \leq \varepsilon_{t f}$, it is the stage I of phase transformation. The phase transformation of the tension side surface material from austenite to martensite occurs. The phase boundary between mixed phase and austenite phase is BTA. As can be seen in Figure 3(b), for $\varepsilon_{c s} \leq\left|\varepsilon_{c}\right| \leq \varepsilon_{c f}$ and $\varepsilon_{t s} \leq \varepsilon_{t} \leq \varepsilon_{t f}$, it is the stage II of phase transformation. The phase transformation of the compression side surface material occurs too. There is a new phase boundary (BCA) between mixed phase and austenite phase on the compression side. In Figure 3(c), for $\varepsilon_{c s} \leq\left|\varepsilon_{c}\right| \leq \varepsilon_{c f}$ and $\varepsilon_{t f} \leq \varepsilon_{t}$, it is the stage III of phase transformation. The surface material of tension side changes into martensite completely. There is a new phase boundary (BTM) between mixed phase and martensite phase on the tension side. As shown in Figure 3(d), for $\varepsilon_{c f} \leq\left|\varepsilon_{c}\right|$ and $\varepsilon_{t f} \leq \varepsilon_{t}$, it is the stage IV of phase transformation. The surface material of compression side changes into martensite completely. There is a new phase boundary (BCM) between mixed phase and martensite phase on the compression side. In Figure 3, the distance of $A, B, C, D$,

$E$ and $F$ to neutral axis are $y_{1}=\left(1+\Delta_{\mathrm{IV}}\right) h, \quad y_{2}=-\left(1-\Delta_{\mathrm{IV}}\right) h, \quad y_{3}=\frac{\varepsilon_{t s}\left(1+\Delta_{\mathrm{IV}}\right) h}{\varepsilon_{t}}, \quad y_{4}=-\frac{\varepsilon_{c s}\left(1+\Delta_{\mathrm{IV}}\right) h}{\varepsilon_{t}}$ $y_{5}=\frac{\varepsilon_{t f}\left(1+\Delta_{\mathrm{IV}}\right) h}{\varepsilon_{t}}$ and $y_{6}=-\frac{\varepsilon_{c f}\left(1+\Delta_{\mathrm{IV}}\right) h}{\varepsilon_{t}}$, respectively. 

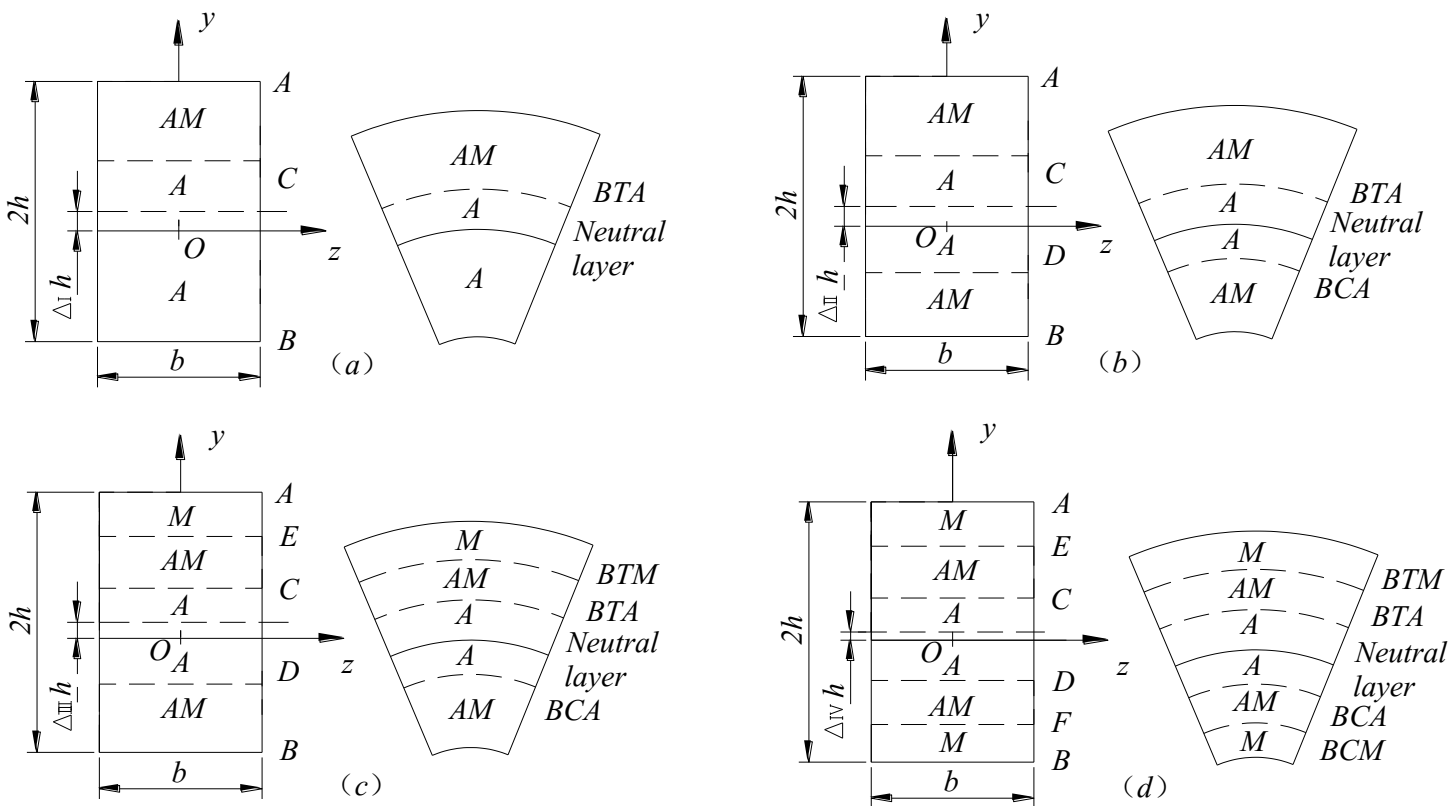

Figure 3: Beam cross section and micro segment bending deformation: (a) I stage of phase transformation, (b) II stage of phase transformation, (c) III stage of phase transformation, (d) IV stage of phase transformation

In the whole process of SMA beam phase transformation, the expression of stress distribution along the height of the cross section can be expressed as Eq. (6). In the stage I of phase transformation, it only meets equation(c) and (d). Now, there is only one phase boundary BTA, and $y_{3}$ is the height from the neutral axis to BTA. In the stage II of phase transformation, it meets equation(b), (c) and (d). At the moment, there are two phase boundaries: BTA and BCA, $y_{3}$ and $y_{4}$ are the height from the neutral axis to BTA and BCA, respectively. In the stage III of phase transformation, it meets equation(b), (c) ,(d)and (e). Meanwhile, there are three phase boundaries: BTA, BCA and BTM, $y_{3}, y_{4}$ and $y_{5}$ are the height from the neutral axis to BTA, $\mathrm{BCA}$ and BTM, respectively. In the stage IV of phase transformation, the expression of stress distribution along the height of the cross section can be written as Eq. (6). At the moment, four phase boundaries(BTA, BCA, BTM and BCM) all occur.

$$
\sigma(y)= \begin{cases}-\sigma_{c f}+E_{M}\left(\frac{y \varepsilon_{t}}{\left(1+\Delta_{i}\right) h}-\varepsilon_{c f}\right), & y_{2} \leq y \leq y_{6} \quad(a) \\ -\sigma_{c s}+E_{1}\left(\frac{y \varepsilon_{t}}{\left(1+\Delta_{i}\right) h}-\varepsilon_{c s}\right), & y_{6} \leq y \leq y_{4} \quad(b) \\ \frac{y E_{A} \varepsilon_{t}}{\left(1+\Delta_{i}\right) h}, & y_{4} \leq y \leq y_{3} \quad(c) \\ \sigma_{t s}+E_{1}\left(\frac{y \varepsilon_{t}}{\left(1+\Delta_{i}\right) h}-\varepsilon_{t s}\right), & y_{3} \leq y \leq y_{5}(d) \\ \sigma_{t f}+E_{M}\left(\frac{y \varepsilon_{t}}{\left(1+\Delta_{i}\right) h}-\varepsilon_{t f}\right), & y_{5} \leq y \leq y_{1} \quad(e)\end{cases}
$$

\subsection{Balance equation}

In the initial stage, the stress distribution is linear, and the bending moment due to stress distribution in the cross section is found as Eq. (7).

$$
\begin{aligned}
M & =\int_{-h}^{h} \sigma(y) y d A \\
& =\int_{-h}^{h} \frac{E_{A} \varepsilon_{t} b y}{h} y d y
\end{aligned}
$$

The stress distribution in the stage I of phase transformation has one linear parts and one transfor- 
mation portions. The bending moment due to this distribution can be found as Eq. (8).

$$
\begin{aligned}
M & =\int_{-h}^{h} \sigma(y) y d A \\
& =\int_{-\left(1-\Delta_{\mathrm{I}}\right) h}^{\frac{\varepsilon_{t s}\left(1+\Delta_{\mathrm{I}}\right) h}{\varepsilon_{\mathrm{I}}}} \frac{E_{A} \varepsilon_{t} b y}{\left(1+\Delta_{\mathrm{I}}\right) h} y d y+\int_{\frac{\varepsilon_{t s}\left(1+\Delta_{\mathrm{I}}\right) h}{\varepsilon_{t}}}^{\left(1+\Delta_{\mathrm{I}}\right) h}\left[\sigma_{t s}+E_{1}\left(\frac{y \varepsilon_{t}}{\left(1+\Delta_{\mathrm{I}}\right) h}-\varepsilon_{t s}\right)\right] d y
\end{aligned}
$$

The stress distribution in the stage II of phase transformation has one linear parts and two transformation portions. The bending moment due to this distribution can be found as Eq. (9).

$$
\begin{aligned}
& M=\int_{-h}^{h} \sigma(y) y d A \\
& =\int_{-\left(1-\Delta_{\mathrm{II}}\right) h}^{-\frac{\varepsilon_{c s}\left(1+\Delta_{\mathrm{II}}\right) h}{\varepsilon_{t}}} b y\left[-\sigma_{c s}+E_{1}\left(\frac{y \varepsilon_{t}}{\left(1+\Delta_{\mathrm{II}}\right) h}-\varepsilon_{c s}\right)\right] d y+\int_{-\frac{\varepsilon_{c s}\left(1+\Delta_{I I}\right) h}{\varepsilon_{I}}}^{\frac{\varepsilon_{t s}\left(1+\Delta_{\mathrm{II}}\right) h}{\varepsilon_{\mathrm{I}}}} \frac{E_{A} \varepsilon_{t} b y}{\left(1+\Delta_{\mathrm{II}}\right) h} y d y \\
& +\int_{\frac{\varepsilon_{I s}\left(1+\Delta_{\mathrm{II}}\right) h}{\varepsilon_{t}}}^{\left(1+\Delta_{\mathrm{II}}\right) h} b y\left[\sigma_{t s}+E_{1}\left(\frac{y \varepsilon_{t}}{\left(1+\Delta_{\mathrm{II}}\right) h}-\varepsilon_{t s}\right)\right] d y
\end{aligned}
$$

The stress distribution in the stage III of phase transformation has one linear parts, two transformation portions and one completed parts of phase transformation. The bending moment due to this distribution can be found as Eq. (10).

$$
\begin{aligned}
& M=\int_{-h}^{h} \sigma(y) y d A \\
& =\int_{-\left(1-\Delta_{\mathrm{III}}\right) h}^{-\frac{\varepsilon_{c s}\left(1+\Delta_{\mathrm{III}}\right) h}{\varepsilon_{t}}} b y\left[-\sigma_{c s}+E_{1}\left(\frac{y \varepsilon_{t}}{\left(1+\Delta_{\mathrm{III}}\right) h}-\varepsilon_{c s}\right)\right] d y+\int_{-\frac{\varepsilon_{c s}\left(1+\Delta_{\mathrm{III}}\right) h}{\varepsilon_{t}}}^{\frac{\varepsilon_{t s}\left(1+\Delta_{\mathrm{III}}\right) h}{\left(1+\Delta_{\mathrm{III}}\right) h}} \frac{E_{A} \varepsilon_{t} b y}{(1 .} \\
& +\int_{\frac{\varepsilon_{t s}\left(1+\Delta_{\text {III }}\right) h}{\varepsilon_{t}}}^{\frac{\varepsilon_{f f}\left(1+\Delta_{\mathrm{III}}\right) h}{\varepsilon_{t}}} b y\left[\sigma_{t s}+E_{1}\left(\frac{y \varepsilon_{t}}{\left(1+\Delta_{\mathrm{III}}\right) h}-\varepsilon_{t s}\right)\right] d y+\int_{\frac{\varepsilon_{t f}\left(1+\Delta_{\mathrm{III}}\right) h}{\varepsilon_{t}}}^{\left(1+\Delta_{\mathrm{II}}\right) h} b y\left[\sigma_{t f}+E_{M}\left(\frac{y \varepsilon_{t}}{\left(1+\Delta_{\mathrm{III}}\right) h}-\varepsilon_{t f}\right)\right] d y
\end{aligned}
$$

The stress distribution in the stageIV of phase transformation has one linear parts, two transformation portions and two completed parts of phase transformation. The bending moment due to this distribution can be found as Eq. (11).

$$
\begin{aligned}
M= & \int_{-h}^{h} \sigma(y) y d A \\
= & \int_{-\left(1-\Delta_{\mathrm{IV}}\right) h}^{-\frac{\varepsilon_{c f}\left(1+\Delta_{\mathrm{IV}}\right) h}{\varepsilon_{t}}} b y\left[-\sigma_{c f}+E_{M}\left(\frac{y \varepsilon_{t}}{\left(1+\Delta_{\mathrm{IV}}\right) h}-\varepsilon_{c f}\right)\right] d y \\
& +\int_{-\frac{\varepsilon_{c f}\left(1+\Delta_{\mathrm{IV}}\right) h}{\varepsilon_{t}}}^{-\frac{\varepsilon_{c s}\left(1+\Delta_{\mathrm{IV}}\right) h}{\varepsilon_{t}}} b y\left[-\sigma_{c s}+E_{1}\left(\frac{y \varepsilon_{t}}{\left(1+\Delta_{\mathrm{IV}}\right) h}-\varepsilon_{c s}\right)\right] d y+\int_{-\frac{\varepsilon_{c s}\left(1+\Delta_{\mathrm{IV}}\right) h}{\varepsilon_{t}}}^{\frac{\varepsilon_{t s}\left(1+\Delta_{\mathrm{IV}}\right) h}{\varepsilon_{t}}} \frac{E_{A} \varepsilon_{t} b y}{\left(1+\Delta_{\mathrm{IV}}\right) h} y d y \\
& +\int_{\frac{\varepsilon_{t s}\left(1+\Delta_{\mathrm{IV}}\right) h}{\varepsilon_{t}}}^{\frac{\varepsilon_{t f}\left(1+\Delta_{\mathrm{IV}}\right) h}{\varepsilon_{t}}} b y\left[\sigma_{t s}+E_{1}\left(\frac{y \varepsilon_{t}}{\left(1+\Delta_{\mathrm{IV}}\right) h}-\varepsilon_{t s}\right)\right] d y+\int_{\frac{\varepsilon_{t f}\left(1+\Delta_{\mathrm{IV}}\right) h}{\varepsilon_{t}} b y}^{\left(1+\Delta_{\mathrm{V}}\right) h}\left[\sigma_{t f}+E_{M}\left(\frac{y \varepsilon_{t}}{\left(1+\Delta_{\mathrm{IV}}\right) h}-\varepsilon_{t f}\right)\right] d y
\end{aligned}
$$

Solving Eq. (5)- Eq. (11), the bending moment is found.

\subsection{Beam deflection}

The nonlinear governing equation is derived by the expression of moment balance equation, which can be written as

$$
\frac{d^{2} w}{d x^{2}}=-\left[1+\left(\frac{d w}{d x}\right)^{2}\right]^{3 / 2} \sqrt{\frac{b \int_{-\varepsilon_{c}}^{\varepsilon_{t}} \sigma \varepsilon d \varepsilon}{M}}
$$

\section{MODEL OF CRITICAL STRESS}

The critical stresses that are used are dependent on temperature. This relationship is illustrated in the phase plot shown in Figure 4. Critical stresses of tension side can be simply found from the transformation lines. In this study, substituting the critical stresses of tension side into Eq. (4), the compression side critical stresses are found. 


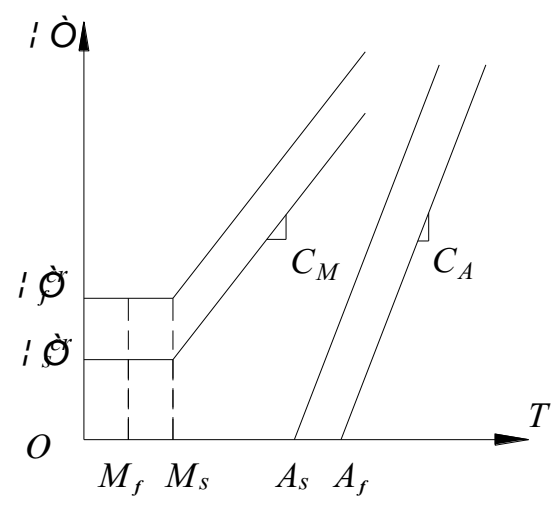

Figure 4: Critical stresses for transformation or martensite twin conversion as functions of temperature

The stresses at the beginning of martensite transformation can be expressed as:

$$
\sigma_{m s}= \begin{cases}\sigma_{s}^{c r}, & T \leq M_{s} \\ \sigma_{s}^{c r}+C_{M}\left(T-M_{s}\right), & T>M_{s}\end{cases}
$$

The stresses at the end of martensite transformation can be expressed as:

$$
\sigma_{m f}= \begin{cases}\sigma_{f}^{c r}, & T \leq M_{s} \\ \sigma_{f}^{c r}+C_{M}\left(T-M_{s}\right), & T>M_{s}\end{cases}
$$

The stresses at the beginning of austenite transformation can be expressed as:

$$
\sigma_{a s}=C_{A}\left(T-A_{s}\right) \quad T>A_{s}
$$

The stresses at the end of austenite transformation can be expressed as:

$$
\sigma_{a f}=C_{A}\left(T-A_{f}\right) \quad T>A_{f}
$$

$\sigma_{m s}, \sigma_{m f}, \sigma_{a s}$ and $\sigma_{a f}$ are the stresses at the beginning and end of martensite transformation and beginning and end of austenite transformation, respectively. $M_{s}, M_{f}, A_{s}$ and $A_{f}$ are the temperature at the beginning and end of martensite transformation and beginning and end of austenite transformation, respec-

\begin{tabular}{|c|c|c|c|}
\hline Moduli & Transformation & Transformation & Maximum \\
\hline & Temperatures & Constants & Residual Strain \\
\hline$E_{A}=67 \times 10^{3} \mathrm{MPa}$ & $M_{f}=9^{\circ} \mathrm{C} \quad M_{s}=18.4^{\circ} \mathrm{C}$ & $C_{M}=8 \mathrm{MPa} /{ }^{\circ} \mathrm{C} \quad C_{A}=13.8 \mathrm{MPa} /{ }^{\circ} \mathrm{C}$ & $\varepsilon_{L}=0.067$ \\
\hline$E_{M}=26.3 \times 10^{3} \mathrm{MPa}$ & $A_{s}=34.5^{\circ} \mathrm{C} \quad A_{f}=49^{\circ} \mathrm{C}$ & $\sigma_{s}^{c r}=100 \mathrm{MPa}$ & \\
\hline
\end{tabular}
tively. $\sigma_{s}^{c r}$ and $\sigma_{f}^{c r}$ are the beginning and end of critical stresses. $C_{A}$ and $C_{M}$ are constants.

Material properties that are used to get the results are presented in table 1[13].

Table 1: Material properties for simulations

\section{RESULTS AND DISCUSSION}

In this section, the material properties are shown in Table 1. The length, height and width of the SMA beam are $l=400 \mathrm{~mm}, 2 h=50 \mathrm{~mm}$ and $b=30 \mathrm{~mm}$, respectively. As the change of the neutral layer of SMA beam, the $x$ axis is changing (in Figure 5).

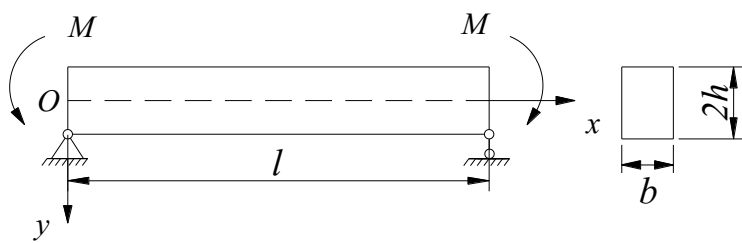

Figure 5: Schematic diagram of SMA simply supported beam 


\subsection{Comparisons with available results}

The validation study of the curvature of the shape memory alloy beam is presented in Figure 6. A shape memory alloy cantilever beam is subjected to a vertical force at the tip. The size and material parameters of the beam are same as the literature [17]. The curvature of the beam at $T=30^{\circ} \mathrm{C}$ and with $F=20 \mathrm{~N}$ during loading is plotted in Figure 6. The results are compared with those available in the literature [17]. When $\alpha=0$, the curves clearly show that the results from this study are close to literature [17]. But when $\alpha \neq 0$, the curvature decreases with the increase of $\alpha$ in the process of shape memory alloys phase transformation.

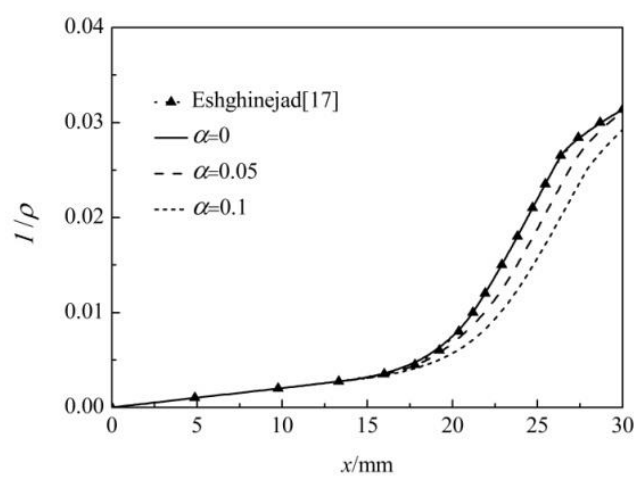

Figure 6: The curvature of shape memory alloy cantilever beam.

\subsection{Neutral axis}

The influence of $\alpha$ on neutral axis offset at $T=60^{\circ} \mathrm{C}$ is shown in Figure 7(a). While the $\alpha=0$, the neutral axis does not gain offset both in initial state and phase transformation stage by an increment of bending moment, and expectedly remain initiated. It can be found that by increasing $\alpha$ the neutral axis offset and the rate of neutral axis moved to the tension side increases. The neutral axis offset is related to the martensitic phase fractions of surface material. When the phase change of the surface material of the tension side occurs, the neutral layer moves to the compression side until the surface material changes into martensite completely. Then, with the increase of bending moment, the neutral layer moves back, even moves to the tension side. The influence of temperature on neutral axis offset at $\alpha=0.05$ is shown in Figure 7(b). The changes of temperature have no effect on the maximal neutral axis offset at the same $\alpha$. By increasing temperature the rate of neutral axis moved to the tension side decreases at the same $\alpha$. In the case of identical scaling description, an increase for temperature will hopefully make curve of neutral axis offset and bending moment move to right. Because the stress at the beginning of phase transformation increases with the increase of temperature. The tension-compression asymmetry is an important characteristic in the process of shape memory alloys phase transformation. Both Eshghinejad et al [17] and Ostadrahimi et al [19] analysis the process of shape memory alloys phase transformation. However, it is noted that some differences between the results of their and this work could be the consequences of neglecting tension-compression asymmetry. The tensioncompression asymmetry make the neutral axis move to the tension side as show in figure 7 . 

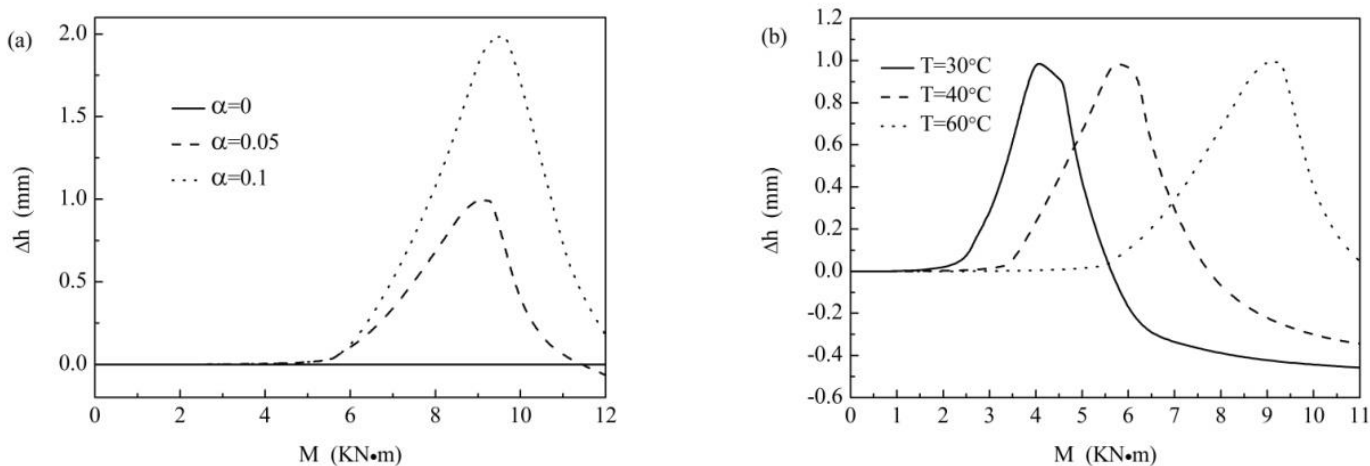

Figure 7: Neutral axis offset

\subsection{Curvature}

The influence of $\alpha$ on curvature at $T=60^{\circ} \mathrm{C}$ is shown in Figure 8(a). In the initial stage, the relationship between curvature and bending moment is linear, but in the phase change stage is nonlinear. The slope of curvature-bending moment curve firstly decreases and then increases. The reason is that the Young's moduli firstly decreases and then increases. With the increase of $\alpha$, the bending moment increases in the case of given curvature. The influence of temperature on curvature at $\alpha=0.05$ is shown in Figure 8(b). In the initial stage, the relationship between curvature and bending moment is linear too. With the increase of temperature, the bending moment increases in the case of given curvature. The temperature has a great influence on curvature subjected to bending moment.
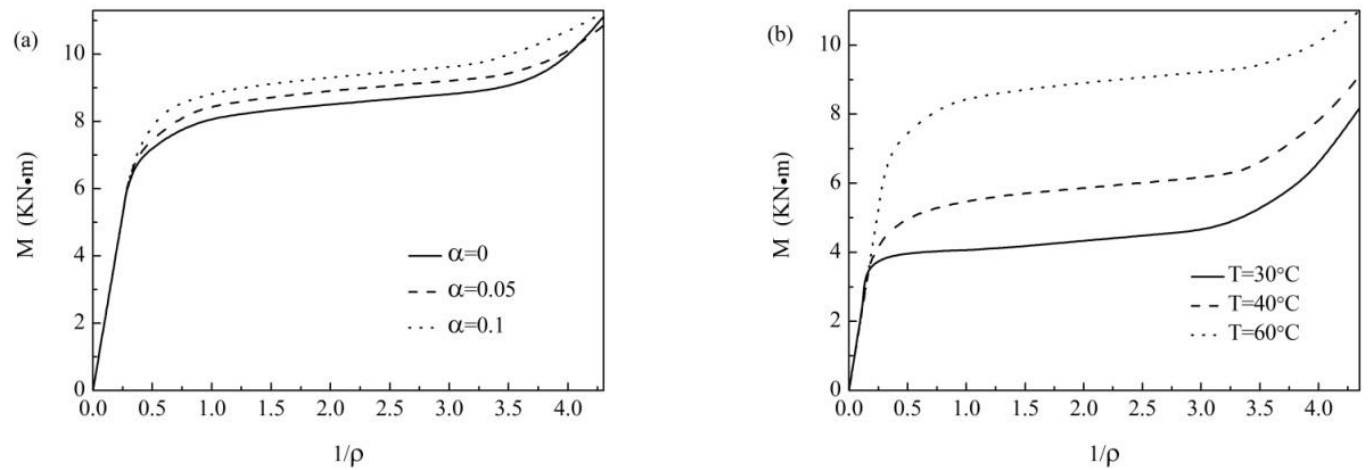

Figure 8: The relationship between curvature and bending moment.

\subsection{Phase boundaries}

Figure 9 illustrates the influence of both $\alpha$ and temperature on the movement of phase boundaries. The influence of $\alpha$ on movement of phase boundaries at $T=60^{\circ} \mathrm{C}$ is shown in Figure 9(a). The phase boundaries on either side of the cross section present symmetry at $\alpha=0$, the phase boundaries obviously present asymmetry at $\alpha \neq 0$. The $\alpha$ has a greater influence on the compression side. The influence of temperature on movement of phase boundaries at $\alpha=0.05$ is shown in Figure 9(b). In this case by increasing temperature the curve slope of relationship between phase boundaries and bending moment decrease. The temperature has a greater influence on tension side and compression side. It can be found that the phase boundaries movementbending moment curve will move to right as the increase of temperature and $\alpha$. It means that the phase transformation of SMA beam become more and more difficult. 

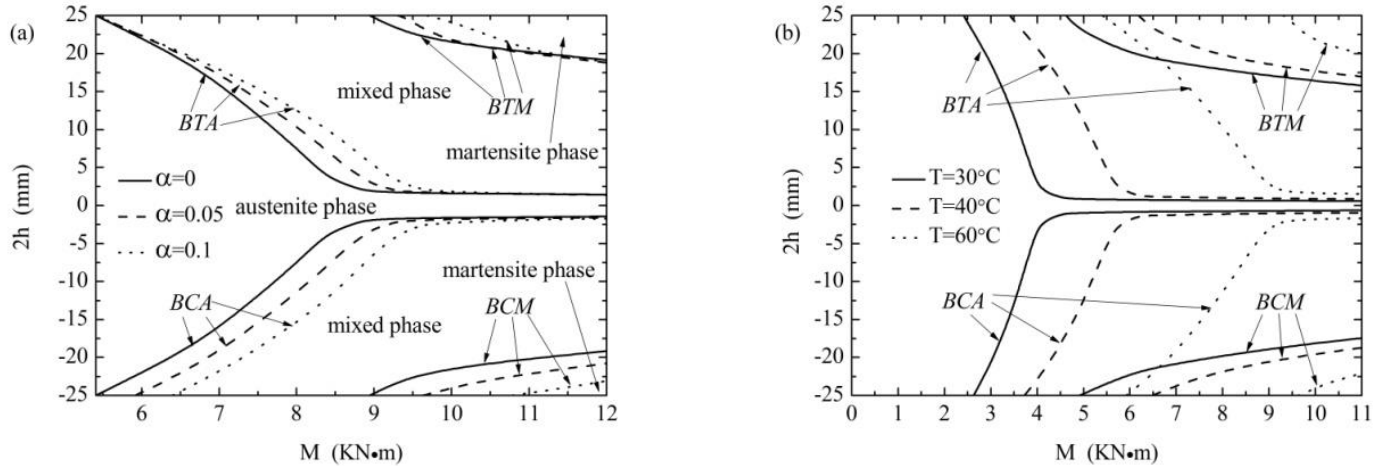

Figure 9: The movement of phase boundaries.

\subsection{Mid-span deflection}

Figure 10 illustrates the influence of $\alpha$ and temperature on the mid-span deflection. The mid-span deflection subjected to different $\alpha$ at $T=60^{\circ} \mathrm{C}$ is shown in Figure 10(a). As can be seen by increasing $\alpha$ the mid-span deflection decreases. Under the case of constant value for temperature, $\alpha$ contributes none to the value of bending moment when phase change takes place. The mid-span deflection subjected to various temperature at $\alpha=0.05$ is shown in Figure 10(b). As can be seen by increasing temperature the mid-span deflection decreases. In the case of identical scaling description, an increase for temperature and an augmentation on $\alpha$ will hopefully make curve of mid-span deflection and bending moment move to right. If the bending moment is small, the material property is linear, the geometry nonlinear have no effect on deflection deformation of beam. If the bending moment is higher, the material property is nonlinear, calculation results of geometry nonlinear is greater than the geometry linear, the geometry nonlinear has noticeable effect on deflection deformation of SMA beam.
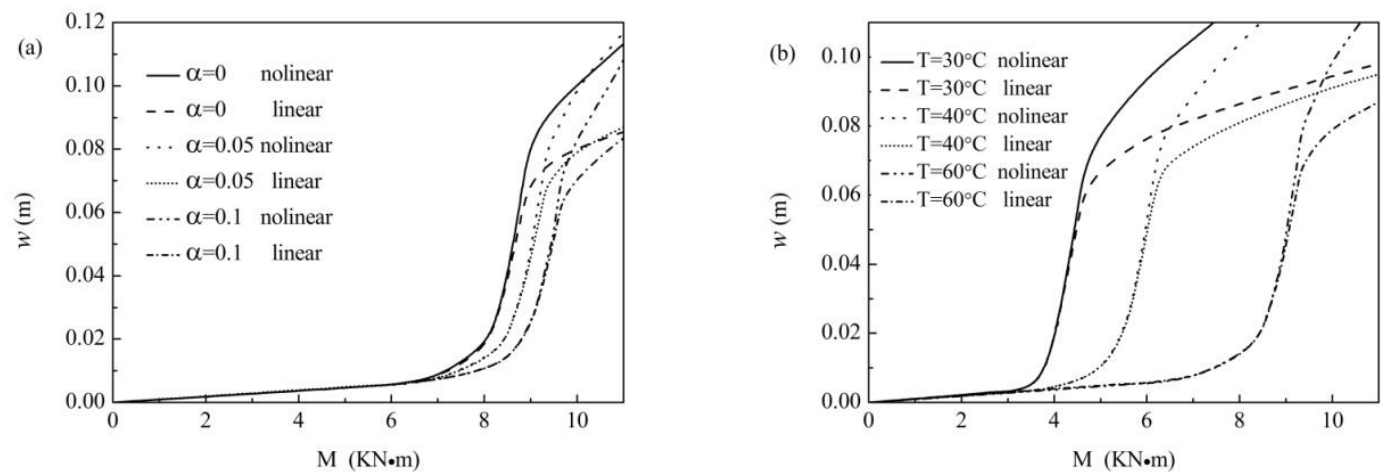

Figure 10: The relationship between mid-span deflection and bending moment.

\subsection{SMA against elastic}

The SMA beam subjected to various bending moments at $T=60^{\circ} C$ and $\alpha=0.05$ and comparison to an elastic beam with $E=E_{A}$ is plotted in Figure 11. In the process of bending deformation, the elastic material has any change and the Young's moduli is always equal to $E_{A}$. As can be seen at $M=5 \mathrm{KN}$, SMA beam deflection and elastic beam deflection are identical, due to that the force was not enough to establish any transformation. With the bending moment increase, more differences between the SMA beam deflection and the elastic beam deflection are observed which is due to increasing the transformation region. Looking into the deflection of the SMA beam and comparing to the elastic beam may be beneficial in engineering point of view, which is due to the displacement of the SMA beam more than elastic beam obviously. SMA beam has superelastic effects. 


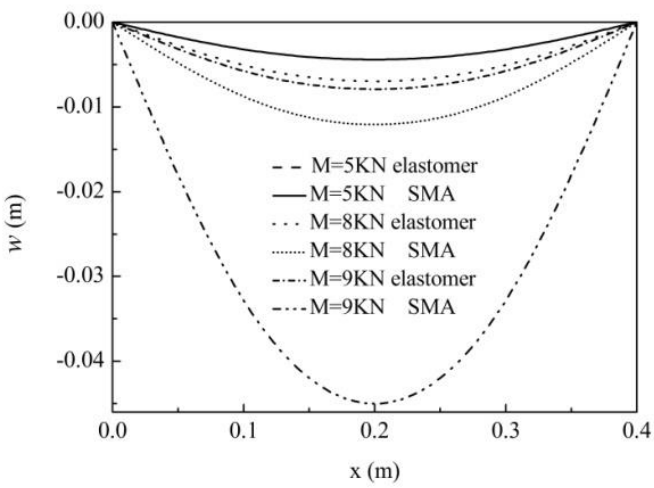

Figure 11: The deflection of SMA beam and elastic beam at $T=60^{\circ} \mathrm{C}$ and $\alpha=0.05$

\section{CONCLUSIONS}

In this paper, a new simple mechanics model is established to study the tension-compression asymmetry of SMA under the thermal and the mechanical loads.

At first, the study focus on solving the different properties between tension and compression of SMA materials, the $\alpha$ is introduced to amend original mechanical model. In the next steps, the split-step method is adopted to analyze the phase transformation of SMA beam. Based on the Euler-Bernoulli beam assumption and the plane assumption, the expression of stress distribution along the height of the cross section is established. The bending moments are found by integration of stress over the cross section for beam. And the curvature can be found by the expression of curvature and strain. These explicit expressions are used for obtaining relations between bending moment and curvature in pure bending. Finally, the displacement of SMA beam is found by double integrating curvature along the beam length. Also, a number of case studies are provided to show the results of the research at different $\alpha$ and temperature.

Studying the tension-compression asymmetry in bending analysis of SMA beam is an important extension of the present work, which can be obtained by using the new simple mechanics model. The approach ends up with the relationship between the bending moment applied to the simply supported beam and the resultant deflection at a specific $\alpha$ and temperature. In addition, the neutral axis offset-bending moment curve, the curvature-bending moment curve and phase boundary-bending moment curve are obtained. The model can be simply and effectively analyzed the tension-compression asymmetry of SMA beam under the thermal and the mechanical loads.

\section{ACKNOWLEDGMENTS}

This work was financially supported by the National Natural Science Foundation of China (No.05-020317).

\section{BIBLIOGRAPHY}

[1] IBRAHIM, M.K., HAMZAH, E., SAUD, S.N., et al., "Microwave sintering effects on the microstructure and mechanical properties of $\mathrm{Ti}-51$ at $\% \mathrm{Ni}$ shape memory alloys", International Journal of Minerals, v. $24, \mathrm{n}$. 3, pp. 280-288, Mar. 2017

[2] LIU, J., HUANG, H., XIE, J., "Effects of aging treatment on the microstructure and superelasticity of columnar-grained Cu71 Al18Mn11 shape memory alloy”, International Journal of Minerals, v. 23, n. 10, pp. 1157-1166, Oct. 2016.

[3] OLIVEIRA, J.P., PANTON, B., ZENG, Z., et al., "Laser welded superelastic Cu-Al-Mn shape memory alloy wires", Materials \& Design, v. 90, n. 1, pp. 122-128, Jan. 2016.

[4] FALUHELYI, P., LEVY-NETO, F., SILVA, E.P., et al., "Thermoelastic Behavior of SMAHC Beams Subjected to Bending Loads at two Temperatures”, Materia (R.J.), v. 18, n. 4, pp. 1491-1500, May. 2013. 
[5] SPEIRS, M., VAN, H.B., VAN, H.J., et al., "Fatigue behaviour of NiTi shape memory alloy scaffolds produced by SLM, a unit cell design comparison", Journal of the Mechanical Behavior of Biomedical Materials, v. 70, n. 1, pp. 53-59, Jan. 2017.

[6] BIFFI, C.A., BASSANI, P., SAJEDI, Z., et al., "Laser ignition in Self-propagating high temperature synthesis of porous NiTinol shape memory alloy", Materials Letters, v. 193, n. 1, pp. 54-57, Apr. 2017.

[7] ABUZAITER, A., NAFEA, M., MOHD, FAUDZI, A.A., et al., "Thermomechanical behavior of bulk NiTi shape-memory-alloy microactuators based on bimorph actuation”, Microsystem Technologies, v. 22, n. 8, pp. 2125-2131, Aug. 2016.

[8] FU, H., LI, W., SONG, S., et al., "Effects of grain orientation and precipitates on the superelasticity in directionally solidified FeNiCoAlTaB shape memory alloy", Journal of Alloys \& Compounds, v. 684, n. 1, pp. 556-563, Nov. 2016.

[9] FU, H., ZHAO, H., SONG, S., et al., "Evolution of the cold-rolling and recrystallization textures in FeNiCoAlNbB shape memory alloy", Journal of Alloys \& Compounds, v. 686, n. 1, pp. 1008-1016, Nov. 2016.

[10] KUMAR, P., JAIN, A.K., HUSSAIN, S., et al., "Changes in the properties of Cu-Al-Mn shape memory alloy due to quaternary addition of different elements", Materia (R.J.), v. 20, n. 1, pp. 284-292, Nov. 2015.

[11] MULLER, I., "A model for a body with shape-memory”, Archive for Rational Mechanics \& Analysis, v. 70, n. 1, pp. 61-77, Mar. 1979.

[12] TANAKA, K., "A Phenomenological Description on Thermomechanical Behavior of Shape Memory Alloys", Journal of pressure vessel technology-transaction of ASME, v. 112, 1990.

[13] LIANG, C., The constitutive modeling of shape memory alloys, 856ed., Virginia, Department of Mechanical Engineering, 1990.

[14] BAJORIA, K.M., KADUSKAR, S.S., "Modeling of a reinforced concrete beam using shape memory alloy as reinforcement bars" Proceedings of SPIE, v. 2, n. 15, pp. 95-102, Apr. 2017.

[15] ATANACKOVIC, T., ACHENBACH, M., "Moment-curvature relations for a pseudoelastic beam Contin”, Continuum Mechanics \& Thermodynamics, v. 1, n. 1, pp. 73-80, Feb. 1989.

[16] FLOR, S.D.L., URBINA, C., FERRANDO, F., “Asymmetrical bending model for NiTi shape memory wires: numerical simulations and experimental analysis: asymmetrical bending model for NiTi SMA" , Strain, v. 47, n. 3, pp. 255-267, Mar. 2011.

[17] ESHGHINEJAD, A., ELAHINIA, M., "Exact Solution for Bending of Shape Memory Alloy Beams", Mechanics of Advanced Materials and Structures, v. 22, n. 10, pp. 829-838, Oct. 2015.

[18] AURICCHIO, F., TAYLOR, R.L., "Shape-memory alloys: modelling and numerical simulations of the finite-strain superelastic behavior", Computer Methods in Applied Mechanics \& Engineering, v. 143, n. 1, pp. 175-194, Apr. 1997.

[19] OSTADRAHIMI, A., ARGHAVANI, J., POORASADION, S., "An analytical study on the bending of prismatic SMA beams”, Smart Materials and Structures, v. 24, n. 12, Sep. 2015.125035.

\section{ORCID}

Jichang Wang

https://orcid.org/0000-0002-6136-4436

Jingning Yang

https://orcid.org/0000-0002-3009-4453 